\title{
Frequency-Domain Turbo Equalisation in Coded SC-FDMA Systems: EXIT Chart Analysis and Performance
}

\author{
Jiayi Zhang, Lie-Liang Yang and Lajos Hanzo \\ School of ECS, University of Southampton, SO17 1BJ, UK. \\ Email: $\{$ jz07r, lly, lh\}@ecs.soton.ac.uk; http://www-mobile.ecs.soton.ac.uk.
}

\begin{abstract}
In this paper, we investigate the achievable performance of channel coded single-carrier frequency-division multiple-access (SCFDMA) systems employing various detection schemes, when communicating over frequency-selective fading channels. Specifically, three types of minimum mean-square error (MMSE) based frequency-domain (FD) turbo equalisers are considered. The first one is the turbo FD linear equaliser (LE). The second one is a parallel interference cancellation (PIC)-assisted turbo FD decision-feedback equaliser (DFE). The final one is the proposed hybrid interference cancellation (HIC)-aided turbo FD-DFE, which combines successive interference cancellation (SIC) with iterative PIC and decoding. The benefit of interference cancellation (IC) is analysed with the EXtrinsic Information Transfer (EXIT) charts. The performance of the coded SC-FDMA systems employing the abovementioned detection schemes is investigated with the aid of simulations. Our studies show that the IC techniques achieve an attractive performance at a moderate complexity.
\end{abstract}

\section{INTRODUCTION}

In time-domain (TD) turbo equalisation schemes [1], such as the maximum a posteriori probability (MAP) soft-in/soft-out (SISO) equaliser, the classic linear equaliser (LE) or the decision-feedback equaliser (DFE) may be invoked, both of which rely on the $a$ priori information provided by the minimum mean-square error (MMSE) detector termed in this paper as 'soft-MMSE'. The $a$ priori information provided for the inner detector/equaliser is represented by a channel interleaved logarithmic-likelihood-ratio (LLR) sequence of the extrinsic information fed back from the outer forward error correction (FEC) decoder. The soft-information is iteratively exchanged between the inner equaliser and the outer FEC decoder, jointly forming a turbo equaliser. Turbo equalisation schemes were analysed using extrinsic information transfer (EXIT) charts in [2], [3].

When considering a high-rate single-carrier frequency-division multiple-access (SC-FDMA) scheme in the context of the 3GPP Long Term Evolution (LTE) system communicating over high-dispersion channels having long channel impulse responses (CIR), the conventional single-tap linear frequency-domain equaliser (FDE) is capable of reducing the computational complexity by employing the discrete and the inverse discrete Fourier transform (DFT/IDFT) operations. Moreover, without joint equalisation and decoding, the FD-LEs or FD-DFEs based on matched-filtering (MF) or MMSE principles typically lead to a residual ISI [4], [5]. In order to efficiently suppress the residual ISI, the above-mentioned SISO turbo equalisation principle may be employed, forming a FD turbo equaliser, referred to here as the turbo FDE.

In [6], [7], various turbo FD-LE schemes conceived for diverse single-carrier multiple-input multiple-output (MIMO) systems were investigated. Rather than using a LE in the FD, the DFE principle relying on interference cancellation (IC), either successive IC (SIC)

\footnotetext{
${ }^{1}$ The work reported in this paper has formed part of the Core 5 Research Programme of the Virtual Center of Excellence in Mobile and Personal Communications, Mobile VCE, www.mobilevce.com, whose funding support, including that of EPSRC, is gratefully acknowledged. Fully detailed technica reports on this research are available to Industrial Members of Mobile VCE.
}

or parallel IC (PIC), may also be adopted for the turbo FDE. In [5], PIC-aided block based iterative FD-DFEs were proposed for the sake of cancelling not only the postcursors, but also the precursors of the ISI. In [8], a reduced complexity algorithm was developed for the above-mentioned FD-DFEs, which achieved the same performance as its higher-complexity benchmark. For the sake of further improving the achievable performance, invoking FEC coding was proposed in [9], [10], where either hard- or soft-decisions may be fed back to the equaliser by the FEC decoder. More recently, the three-stage MMSE-assisted turbo FDE principle was proposed in [11], which includes a SISO FD-LE, a unity-rate inner code and an irregular outer recursive convolutional code. To the best of our knowledge, the beneficial impact of the IC-assisted turbo FD-DFE on the achievable performance of the LTE uplink has not been quantified in the open literature, which motivates our research in this paper.

Specifically, in this paper we investigate the bit-interleaved coded modulation (BICM) [3] aided SC-FDMA uplink employing three different types of turbo FDEs, when communicating over frequencyselective fading channels. The first type is the soft-MMSE based turbo FD-LE [1]. The second type is referred to as the PIC-assisted turbo FD-DFE, which combines iterative PIC with FEC decoding. Finally, our proposed scheme represents the third type, which is referred to as the hybrid IC (HIC)-assisted turbo FD-DFE. In the HIC-assisted turbo FD-DFE, first the MMSE-SIC algorithm [12], [13] is invoked to suppress the ISI. Then the above-mentioned PIC-aided turbo FDDFE is active, in order to further improve the attainable performance. In this paper, the iterative improvement of the mutual information exchanged between the various FDEs and the FEC decoder is quantified with the aid of EXIT charts. The bit error ratio (BER) performance of the SC-FDMA employing the above-mentioned FDEs is studied with the aid of simulations. Our studies show that the softMMSE invoked in the turbo FD-LE is capable of providing a better performance than the iterative PIC relying on the MMSE principle. Furthermore, the attainable BER performance demonstrates that the lower-complexity PIC-aided turbo FD-DFE requires $0-3 \mathrm{~dB}$ higher uplink transmit power, in comparison to the turbo FD-LE, when both of them use a relatively low number of iterations. Additionally, the HIC-aided turbo FD-DFE may achieve a higher maximum rate compared to the PIC-aided FD-DFE. Ultimately, all the IC techniques considered strike an attractive trade-off between the BER achieved and the complexity imposed.

\section{SYSTEM MODEL}

\section{A. Transmitted Signal}

The BICM-aided SC-FDMA transmitter is shown in Fig. 1. Specifically, at the $k$-th mobile terminal (MT) $(k=0,1, \cdots, K-1)$ the $N_{\mathrm{b}}$-length binary source data stream $\boldsymbol{b}_{k}^{\mathrm{o}}$ is initially encoded by an outer channel code, which in this paper is either a Recursive Systematic Convolutional (RSC) code [3] or the 'turbo-like' Irregular Repeat-Accumulate (IrRA) code of [14], having a coding rate of $R_{\mathrm{c}}$. Then, the outer encoded bit stream $\boldsymbol{c}_{k}^{\mathrm{o}}$ of Fig. 1 is interleaved 


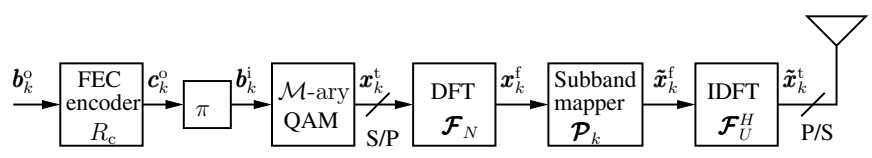

Fig. 1. Transmitter block diagram for BICM aided SC-FDMA

by a $N_{\mathrm{c}}$-length random bit-wise interleaver $\pi$ and its output bit stream $\boldsymbol{b}_{k}^{\mathrm{i}}=\left[\left(\boldsymbol{b}_{k, 0}^{\mathrm{i}}\right)^{T},\left(\boldsymbol{b}_{k, 1}^{\mathrm{i}}\right)^{T}, \cdots,\left(\boldsymbol{b}_{k,\left(N_{\mathrm{s}}-1\right)}^{\mathrm{i}}\right)^{T}\right]^{T}$ is fed into the $\mathcal{M}$-ary set-partition (SP) labelled quadrature amplitude modulation (QAM) mapper transmitting $Q$ bits per symbol, where we have $N_{\mathrm{s}}=N_{\mathrm{c}} / Q$ and $\mathcal{M}=2^{Q}$. Hence, we partition the $N_{\mathrm{c}}$-length bit sequence $\boldsymbol{b}_{k}^{\mathrm{i}}$ of Fig. 1 into $N_{\mathrm{s}}$ segments $\boldsymbol{b}_{k, n_{\mathrm{s}}}^{\mathrm{i}}$, each having a length of $Q$, for $n_{\mathrm{s}}=0,1, \cdots, N_{\mathrm{s}}-1$. Moreover, the $N_{\mathrm{s}}$-length modulated symbol sequence of Fig. 1 is converted to $N_{\mathrm{v}}$ symbol vectors $\boldsymbol{x}_{k}^{\mathrm{t}}\left[n_{\mathrm{v}}\right]\left(n_{\mathrm{v}}=0,1, \cdots, N_{\mathrm{v}}-1\right)$, where each vector $\boldsymbol{x}_{k}^{\mathrm{t}}\left[n_{\mathrm{v}}\right]$ constitutes a SC-FDMA symbol, containing $N$ symbol elements $x_{k, n}^{\mathrm{t}}\left[n_{\mathrm{v}}\right](n=0,1, \cdots, N-1)$ in the TD that are transmitted simultaneously by the user $k$.

Furthermore, as shown in Fig. 1, the SC-FDMA symbol is transformed by an $N$-point DFT [15] from the TD to the FD, yielding $\boldsymbol{x}_{k}^{\mathrm{f}}=\mathcal{F}_{N} \boldsymbol{x}_{k}^{\mathrm{t}}$, where $\mathcal{F}_{N}$ denotes the normalised $N$-point DFT matrix. The $k$-th user's resultant symbols are then mapped to the $N$ consecutive subbands selected from the entire set of $U=(M \times N)$ subbands with the aid of the matrix $\boldsymbol{P}_{k}$ for MT $k$, forming the so-called localised FDMA (LFDMA) scheme [15]. Specifically, we have the $(u, n)$-th entry of $\mathcal{P}_{k}$ defined by $\mathcal{P}_{k,(u, n)}=1$ if $u=$ $k N+n$, otherwise $\mathcal{P}_{k,(u, n)}=0$, for $n=0,1, \cdots, N-1$ and $u=0,1, \cdots, U-1$. The resultant $U$-element FD symbol vector is then transformed to the TD by the $U$-point IDFT, which is similar to the action of the OFDM transmitter [15]. Consequently, the $U$ symbol baseband-equivalent discrete-time signal $\boldsymbol{s}_{k}^{\mathrm{t}}$ transmitted by the $k$-th MT before inserting the cyclic-prefix (CP) can be expressed as

$$
\boldsymbol{s}_{k}^{\mathrm{t}} \quad=\sqrt{P_{k}} \mathcal{F}_{U}^{H} \mathcal{P}_{k} \mathcal{F}_{N} \boldsymbol{x}_{k}^{\mathrm{t}},
$$

where the superscript ${ }^{\mathrm{t}}$ refers to the TD signal. We assume that the transmit power is normalised to unity, yielding $P_{k}=1$ in Eq. (1). In Eq. (1), $\mathcal{F}_{U}^{H}$ denotes the normalised $U$-point IDFT matrix.

\section{B. Signal Representation at the BS Receiver}

The $U$-element TD signal vector holding all the $K$ users' data received at the BS may be expressed as:

$$
\tilde{\boldsymbol{y}}^{\mathrm{t}}=\sum_{k=0}^{K-1} \tilde{\boldsymbol{H}}_{k}^{\mathrm{t}} \boldsymbol{s}_{k}^{\mathrm{t}}+\tilde{\boldsymbol{n}}^{\mathrm{t}}
$$

after removing the $\mathrm{CP}$, where $\tilde{\boldsymbol{H}}_{k}^{\mathrm{t}}$ denotes the $(U \times U)$-element TD circulant channel coefficient matrix of the $k$-th user. Next, after the $U$-point DFT transforming the signal $\tilde{\boldsymbol{y}}^{\mathrm{t}}$ into the FD, the $k^{\prime}$-th user's signal is then demapped into the appropriate subbands according to the subband allocation represented by $\mathcal{P}_{k^{\prime}}$, yielding

$$
\boldsymbol{y}_{k^{\prime}}^{\mathrm{f}}=\boldsymbol{\mathcal { P }}_{k^{\prime}}^{T} \mathcal{F}_{U}\left(\sum_{k=0}^{K-1} \tilde{\boldsymbol{H}}_{k}^{\mathrm{t}} \boldsymbol{s}_{k}^{\mathrm{t}}+\tilde{\boldsymbol{n}}^{\mathrm{t}}\right)=\boldsymbol{H}_{k^{\prime}} \boldsymbol{x}_{k^{\prime}}^{\mathrm{t}}+\boldsymbol{n}_{k^{\prime}}^{\mathrm{f}}
$$

where $\boldsymbol{H}_{k^{\prime}}=\boldsymbol{\mathcal { P }}_{k^{\prime}}^{T} \mathcal{F}_{U} \tilde{\boldsymbol{H}}_{k}^{\mathrm{t}} \mathcal{F}_{U}^{H} \boldsymbol{\mathcal { P }}_{k^{\prime}} \mathcal{F}_{N}$ represents the equivalent dispersive FD channel matrix, which is not exactly a diagonal matrix due to the residual ISI still contaminating the symbols after MMSE equalisation. In Eq. (3), $\boldsymbol{n}_{k^{\prime}}^{\mathrm{f}}=\boldsymbol{\mathcal { P }}_{k^{\prime}}^{T} \mathcal{F}_{U} \tilde{\boldsymbol{n}}^{\mathrm{t}}$ is the noise imposed at the BS's receiver, each element of which has a power of $\mathcal{N}_{n}=\sigma_{\mathrm{N}}^{2}$.

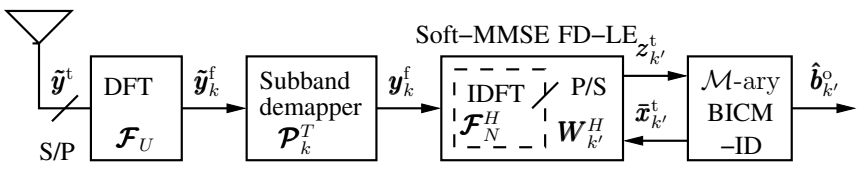

(a) SC-FDMA turbo FD-LE Receiver

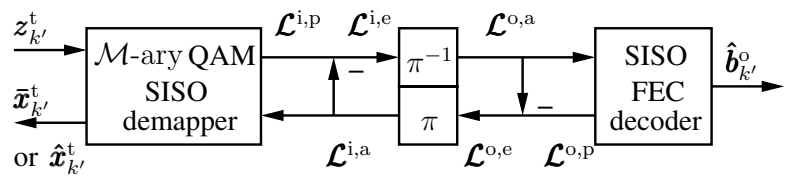

(b) BICM using iterative decoding

Fig. 2. Schematic diagram of turbo receiver for SC-FDMA

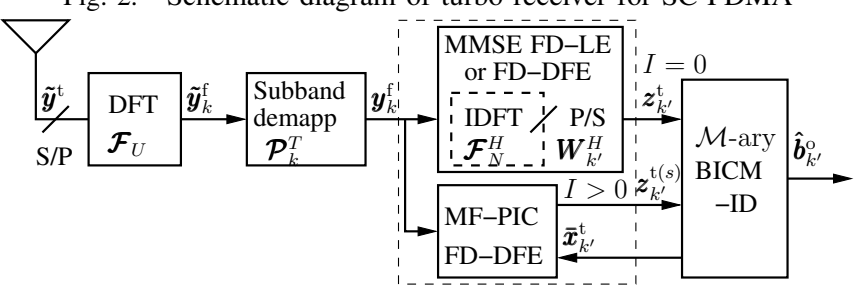

Fig. 3. PIC or HIC aided turbo FD-DFE receiver for SC-FDMA

\section{Frequency-Domain Turbo Equalisation}

\section{A. Soft-MMSE based Turbo FD-LE}

The BS's receiver conceived for BICM-aided SC-FDMA systems is shown in Fig. 2(a), which invokes the complex-valued MMSE turbo FD-LE using the iterative decoding (ID) scheme shown in Fig. 2(b). The corresponding iterative equalisation and decoding philosophy was detailed in [3]. As shown in Fig. 2, following the initial equalisation by the classic MMSE FD-LE and the BICM decoding, these blocks exchange extrinsic information in a number of consecutive iterations, in order to improve the attainable performance. To elaborate a little further, the mean of $\boldsymbol{x}_{k^{\prime}}^{\mathrm{t}}$, denoted by $\boldsymbol{x}_{k^{\prime}}^{\mathrm{t}}$ in Fig. 2(a), is generated by the bit-probability-to-symbol-probability conversion using a-priori LLRs, which are generated by appropriately ordering them by the interleaver based on the feedback of the BICM decoder seen in Fig. 2(b). Therefore, by applying soft-information exchange between the equaliser and BICM decoder, the weight matrix of the MMSE FD-LE is updated based on the a-priori information gleaned during each iteration, which results in a high complexity. We refer this regime as soft-MMSE which further reduces the residual ISI imposed on the estimated symbols, but the required number of iterations may be increased. The design philosophy of the MMSE turbo FD-LE receiver was detailed in [1].

\section{B. IC Assisted Turbo FD-DFE}

Apart from the soft-MMSE aided turbo FD-LE, the turbo FD-DFE may be considered as an alternative technique of mitigating multipath fading. The multi-stage PIC [15] aided turbo FD-DFE of Fig. 3 may employ a LE based on the MMSE principle, which is termed as the linear MMSE (L-MMSE), invoked for the initial filtering of the received signal in the FD, before FEC decoding takes place. Then, this PIC aided turbo FD-DFE carries out the MF based PIC (MF-PIC) operation on a block-by-block basis using the FEC decoded bit stream during each iteration. However, the residual ISI persisting after the initial LE process may degrade the performance of the FEC decoder and the resultant error propagation may even be further precipitated during the PIC phase, unless additionally turbo iterations are invoked.

The real-valued MMSE based FD-DFE [13] relying on the SIC principle is portrayed in Figure 4, which is referred to as the 


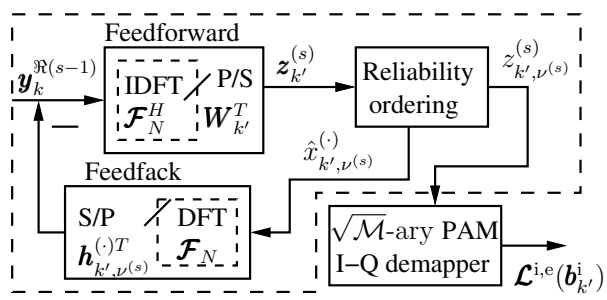

Fig. 4. Reliability-aided real-valued MMSE-SIC [13]

MMSE-SIC FD-DFE scheme. The related reliability-aided MMSESIC philosophy was originally applied for detection in CDMA or SDMA systems [12], [13]. Unlike the conventional MMSE-SIC, which quantifies the reliabilities of the detected symbols in terms of the SINR, the real-valued MMSE-SIC determines the SIC-ordering based on the maximum MAP criterion. Therefore, the impact of both the fading and that of random noise is taken into account during the SIC process.

Rather than using the FD-LE for initial equalisation, our proposed HIC-aided turbo FD-DFE scheme invokes the MMSE-SIC in order to make the symbols as reliable as possible for decoding during the first phase. Then, this MMSE-SIC operation is followed by the PIC based turbo FD-DFE which is combined with the BICM-ID decoding step. As shown in Fig. 3, following the MMSE-SIC based FD-DFE, the extrinsic information output by the decoder is fed forward as the $a$ priori LLR of the first-stage PIC, rather than being fed back as that of the soft-MMSE equaliser of Fig. 2(a). Therefore, the soft-information generated by the PIC scheme may be iteratively exchanged between the MF-PIC based FD-DFE and the FEC decoder. By incorporating the real-valued MMSE-SIC shown in Figure 4 into the Type-II turbo FDE of our SC-FDMA, we are now capable of removing nearly all the residual ISI. Let us briefly elaborate on these procedures as follows.

1) MMSE-SIC: Based on Eq. (3), the equivalent real-valued $2 N$ element signal model of $\boldsymbol{y}_{k^{\prime}}^{\Re}=\boldsymbol{H}_{k^{\prime}}^{\Re} \boldsymbol{x}_{k^{\prime}}^{\Re}+\boldsymbol{n}_{k^{\prime}}^{\Re}$ may be formed, where $\boldsymbol{H}_{k^{\prime}}^{\Re}$ denotes a $(2 N \times 2 N)$-element real-valued version of $\boldsymbol{H}_{k^{\prime}}$ [13]. Thus, the $n$-th element of the real or imaginary part denoted as $(\cdot)$ part in the estimated TD vector $\boldsymbol{z}_{k^{\prime}}^{\Re}$ may be derived as

$$
z_{k^{\prime}, n}^{(\cdot)}=\boldsymbol{w}_{k^{\prime}, n}^{(\cdot) T} \boldsymbol{h}_{k^{\prime}, n}^{(\cdot)} x_{k^{\prime}, n}^{(\cdot)}+\boldsymbol{w}_{k^{\prime}, n}^{(\cdot) T}\left[\boldsymbol{H}_{k^{\prime}}^{\Re} \boldsymbol{x}_{k^{\prime}}^{\Re}-\boldsymbol{h}_{k^{\prime}, n}^{(\cdot)} x_{k^{\prime}, n}^{(\cdot)}+\hat{\boldsymbol{n}}_{k^{\prime}}^{\Re}\right],
$$

where $\boldsymbol{h}_{k^{\prime}, n}^{(\cdot)}$ is the $n$-th column of the (·)-part of $\boldsymbol{H}_{k^{\prime}}^{\Re}$. Furthermore, $\boldsymbol{w}_{k^{\prime}, n}$ represents the $n$-th column of the (.)-part in the MMSE weightmatrix $\boldsymbol{W}_{k^{\prime}}^{\Re}$ expressed as [13]

$$
\boldsymbol{w}_{k^{\prime}, n}^{(\cdot)}=\boldsymbol{H}_{k^{\prime}}^{\Re}\left[\left(\boldsymbol{H}_{k^{\prime}}^{\Re}\right)^{T} \boldsymbol{H}_{k^{\prime}}^{\Re}+\sigma_{\mathrm{N}}^{2} \boldsymbol{I}_{2 N}\right]^{-1} \boldsymbol{e}_{n}^{(\cdot)}
$$

where $\boldsymbol{e}_{n}^{(\cdot)}$ denotes a vector in which the $i$-th element is 0 for $(i \neq n)$, while the $n$-th element of the $(\cdot)$-part is 1 .

Moreover, the detection reliability of $x_{k^{\prime}, n}^{\mathrm{I}}\left(\right.$ or $\left.x_{k^{\prime}, n}^{\mathrm{Q}}\right)$ of $\boldsymbol{x}_{k^{\prime}}^{\Re}$ based on the MAP criterion has to be estimated for determining the SICordering, which is formulated as

$$
p_{n}^{\mathrm{i}, \mathrm{p}(\cdot)}=\frac{\max _{\mathrm{s}_{i^{\prime}} \in \mathcal{S}^{\prime}}\left\{\exp \left(-\varrho_{n, i^{\prime}}^{(\cdot)}+p_{n, i^{\prime}}^{\mathrm{i}, \mathrm{a}(\cdot)}\right)\right\}}{\sum_{\mathrm{s}_{j^{\prime}} \in \mathcal{S}^{\prime}} \exp \left(-\varrho_{n, j^{\prime}}^{(\cdot)}+p_{n, j^{\prime}}^{\mathrm{i}, \mathrm{a}(\cdot)}\right.}
$$

where $p_{n, i^{\prime}}^{\mathrm{i}, \mathrm{a}(\cdot)},\left(i^{\prime}, j^{\prime}=0,1, \cdots, \sqrt{\mathcal{M}}-1\right)$, are the a-priori probabilities of the real part or imaginary part of the symbols. For the $i^{\prime}$-th $\sqrt{\mathcal{M}}$-ray PAM symbols associated with half-power in the constellation set $\mathcal{S}^{\prime}$, we have $\varrho_{n, i^{\prime}}^{(\cdot)}=\left|z_{k^{\prime}, n}^{(\cdot)}-\mu_{n, i^{\prime}}^{(\cdot)}\right|^{2} / \sigma_{n, i^{\prime}}^{(\cdot) 2}$, where $\mu_{n, i^{\prime}}^{(\cdot)}$ and $\sigma_{n, i^{\prime}}^{(\cdot) 2}$ represent the mean and variance of $z_{k^{\prime}, n}^{(\cdot)}$, respectively.
The $\iota$-th element of $\mathcal{L}^{\mathrm{i}, \mathrm{e}}\left(\boldsymbol{b}_{k^{\prime}}^{\mathrm{i}}\right)$ may be represented by the $(n Q+q)$ th elements of the $n_{\mathrm{v}}$-th segment in $\mathcal{L}^{\mathrm{i}, \mathrm{e}}\left(\boldsymbol{b}_{k^{\prime}}^{\mathrm{i}}\right)$, which is the corresponding soft-output extrinsic information of the MMSE-SIC FDDFE expressed as:

$$
\mathcal{L}_{k^{\prime}, \iota}^{\mathrm{i}, \mathrm{e}}=\ln \frac{\sum_{\forall \mathbf{b}_{i^{\prime}}: \mathrm{b}_{\boldsymbol{\prime}_{i, q^{\prime}}=0}} \exp \left(-\varrho_{n_{\mathrm{s}}, i^{\prime}}\right)}{\sum_{\forall \mathbf{b}_{i^{\prime}}: \mathrm{b}_{i^{\prime}, q^{\prime}}=1} \exp \left(-\varrho_{n_{\mathrm{s}}, i^{\prime}}\right)},
$$

while for the $n$-th symbol, we have

$$
\iota= \begin{cases}\left(n_{\mathrm{v}} N+n\right) Q+q^{\prime}+Q / 2, & \text { real part } \\ \left(n_{\mathrm{v}} N+n\right) Q+q^{\prime}, & \text { imaginary part }\end{cases}
$$

respectively. Additionally, we have $n_{\mathrm{s}}=n_{\mathrm{v}} N+n$ for $n_{\mathrm{s}}=$ $0,1, \cdots, N_{\mathrm{s}}-1, n=0,1, \cdots, N-1$ and $q^{\prime}=0,1, \cdots, Q / 2-1$. In Eq. (7), the $Q$-element vector $\mathbf{b}_{i^{\prime}}$ of bits denotes the bit pattern of the $i^{\prime}$-th symbol in the $\sqrt{\mathcal{M}}$-ary constellation symbol alphabet $\mathcal{S}^{\prime}$ and the $q^{\prime}$-th element of $\mathbf{b}_{i^{\prime}}$ corresponds to a symbol $\dot{b}_{i^{\prime}, q^{\prime}}=+1$, for $\mathrm{b}_{i, q}=0$, and $\mathrm{b}_{i^{\prime}, q^{\prime}}=-1$ for $\mathrm{b}_{i, q}=1$.

According to [12], during each SIC stage $s$ for $s=$ $0,1, \cdots,(2 N-1)$, we update $\boldsymbol{z}_{k^{\prime}}^{(s)}=\left(\boldsymbol{W}_{k^{\prime}}^{(s-1)}\right)^{T} \boldsymbol{y}_{k^{\prime}}^{(s-1)}$. Then the index of the most reliable symbol in the set $\breve{\mathcal{N}}$ is obtained by evaluating $\nu^{(s)}=\arg \max _{n^{\prime} \in \mathcal{N}}\left\{p_{n^{\prime}}^{\mathrm{i}, \mathrm{p}(\cdot)}\right\}$. By making a decision based on $z_{k^{\prime}, \nu(s)}^{(s)}$, we obtain the corresponding real or imaginary part of a symbol $\hat{x}_{k^{\prime} \nu^{(s)}}^{(\cdot)}$, which is used for SIC by invoking the operation of $\boldsymbol{y}_{k^{\prime}}^{(s)}=\boldsymbol{y}_{k^{\prime}}^{(s-1)}-\boldsymbol{h}_{k^{\prime}, \nu(s)}^{(\cdot)} \hat{x}_{k^{\prime}, \nu^{(s)}}^{(\cdot)}$. Hence, we have the following MMSE weight matrix at the $s$-th stage [13]

$$
\boldsymbol{W}_{k^{\prime}}^{(s)}=\left[\boldsymbol{W}_{k^{\prime}}^{(s-1)}+\frac{\boldsymbol{w}_{k^{\prime}, \nu^{(s)}}^{(s-1)} \boldsymbol{h}_{k^{\prime}, \nu}^{(\cdot) T} \boldsymbol{W}_{k^{\prime}}^{(s-1)}}{1-\boldsymbol{h}_{k^{\prime}, \nu}^{(\cdot) T} \boldsymbol{w}_{k^{\prime}, \nu^{(s)}}^{(s-1)}}\right] \boldsymbol{\Xi}_{2 N}^{(s)},
$$

where $\boldsymbol{\Xi}_{2 N}^{(s)}$ is a permutation matrix obtained from $\boldsymbol{I}_{2 N}$ by deleting the columns corresponding to the symbols that have already been detected.

2) Iterative MF-PIC: After the random soft-bit interleaver $\pi$, the resultant a-priori LLRs are used for making a decision concerning the $n_{\mathrm{s}}$-th QAM symbol in the received frame based on the MAP criterion for $n_{\mathrm{s}}=0,1, \cdots, N_{\mathrm{s}}-1$. The soft-estimates of the PIC aided DFE may be expressed as

$$
\bar{x}_{k^{\prime}, n}^{\mathrm{t}}=\sum_{\mathrm{s}_{i} \in \mathcal{S}} \mathrm{s}_{i} \cdot \prod_{q=0}^{Q-1} \frac{1}{2}\left(1+\dot{\circ}_{i, q} \tanh \frac{\mathcal{L}_{k^{\prime},\left(n_{\mathrm{s}} Q+q\right)}^{\mathrm{i}, \mathrm{a}}}{2}\right) .
$$

Based on Eq. (3), at the $s$-th PIC stage for $s=1,2, \cdots$, the interference imposed by all the estimated symbols - except for the $n$-th symbol - is then suppressed according to [15]

$$
\boldsymbol{y}_{k^{\prime}, n}^{\mathrm{f}(s)}=\boldsymbol{y}_{k^{\prime}}^{\mathrm{f}}-\boldsymbol{H}_{k^{\prime}} \boldsymbol{\Xi}_{n} \overline{\boldsymbol{x}}_{k^{\prime}}^{\mathrm{t}(s-1)}
$$

where $\Xi_{n}$ is the permutation matrix generated by nulling the $n$ th diagonal element in the matrix $\boldsymbol{I}_{N}$. Therefore, the $n$-th decision variable generated after the $s$-th PIC stage becomes available by carrying out the MF operation, yielding:

$z_{k^{\prime}, n}^{\mathrm{t}(s)}=\boldsymbol{h}_{k^{\prime}, n}^{H} \boldsymbol{h}_{k^{\prime}, n} x_{k^{\prime}, n}^{\mathrm{t}}+\boldsymbol{h}_{k^{\prime} n}^{H} \boldsymbol{H}_{k^{\prime}} \boldsymbol{\Xi}_{n}\left(\boldsymbol{x}_{k^{\prime}}^{\mathrm{t}}-\overline{\boldsymbol{x}}_{k^{\prime}}^{\mathrm{t}(s-1)}\right)+\boldsymbol{h}_{k^{\prime}, n}^{H} \boldsymbol{n}_{k^{\prime}}^{\mathrm{f}}$.

The remaining ISI is further suppressed during each iteration between the PIC aided FD-DFE and the BICM decoder by reducing the estimation errors of $\boldsymbol{x}_{k^{\prime}}^{\mathrm{t}}-\overline{\boldsymbol{x}}_{k^{\prime}}^{\mathrm{t}(s-1)}$. Finally, the mean and the variance of $z_{k^{\prime}, n}^{\mathrm{t}(s)}$ are used for calculating the extrinsic LLRs for the next iteration. 
TABLE I

SIMULATION PARAMETERS

\begin{tabular}{l||c}
\hline BICM-1 & 1/2-rate RSC $(2,1,3)$ coded 4-QAM (SP); \\
BICM-2 & 1/4-rate NS-IrRA $(4,1,10)$ coded 16-QAM (SP) \\
\hline $\begin{array}{l}\text { MMSE } \\
\text { turbo FDE }\end{array}$ & (3) Turbo FD-LE; (2) PIC-aided turbo FD-DFE; \\
\hline
\end{tabular}

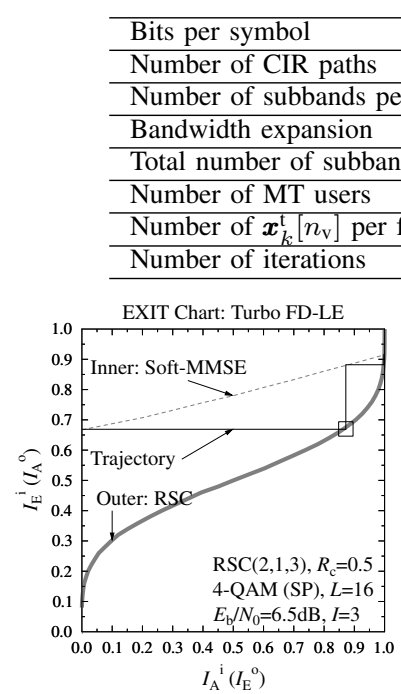

(a) BICM-1

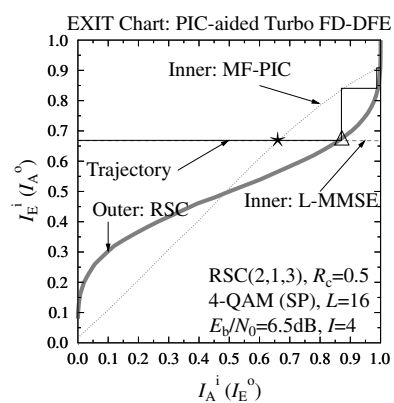

(c) BICM-1

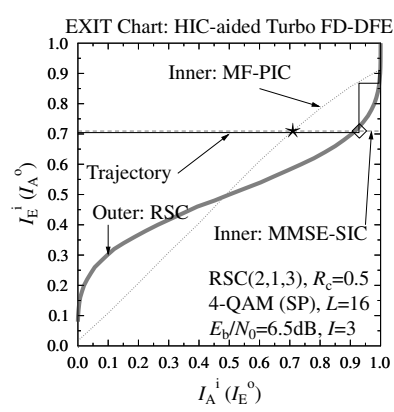

(e) BICM-1

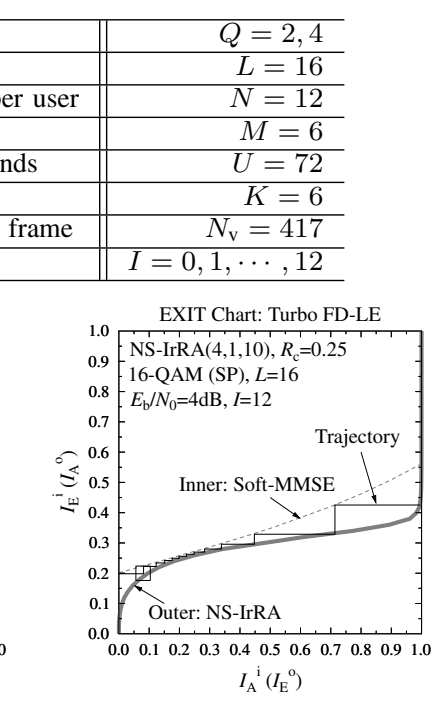

(b) BICM-2

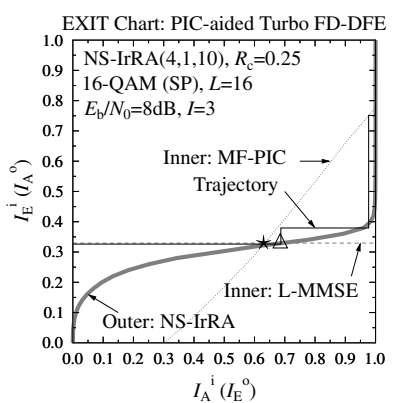

(d) BICM-2

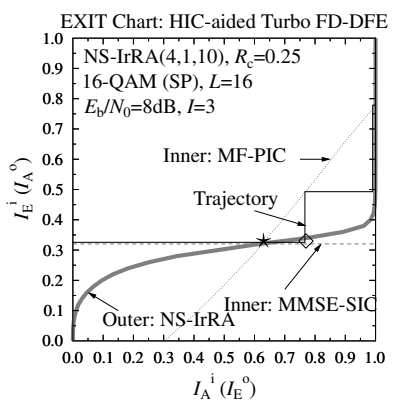

(f) $\mathrm{BICM}-2$
Fig. 5. EXIT charts of the various MMSE turbo FDEs schemes.

\section{Simulation Results And Discussions}

In this section, we evaluate the performance achieved by the various turbo FDEs schemes relying on the LFDMA system for direct transmission to the single-antenna aided BS over uncorrelated frequency-selective Rayleigh fading channels. We assume perfect power control, which eliminates the effects of shadowing. Our simulation parameters are summarised in Table I.

Fig. 5 portrays the EXIT charts of three types of MMSE based turbo FDE schemes invoking two different BICM arrangements to be detailed later for the LFDMA systems, respectively. ${ }^{2}$ The turbo FD-LE scheme of Fig. 2(a) produces similar extrinsic values of $\mathcal{I}_{\mathrm{E}}^{\mathrm{i}}$ to those of the MMSE FD-LE (L-MMSE curves in Fig. 5(a) and 5(c)) for $\mathcal{I}_{\mathrm{A}}^{\mathrm{i}}=0$, when operating at a given $E_{\mathrm{b}} / N_{0}$. Then the $\mathcal{I}_{\mathrm{E}}^{\mathrm{i}}$ value provided by the turbo FD-LE scheme is monotonically increased as a function of $\mathcal{I}_{\mathrm{A}}^{\mathrm{i}}$ as shown in both Fig. 5(a) and 5(b) for BICM1 and BICM-2 schemes, respectively. Moreover, observe in Fig. 3 for the PIC-aided turbo FD-DFE and for the HIC-aided turbo FDDFE arrangements that the a-priori information is only exploited by the MF-PIC FD-DFE block of Fig. 3. Observe furthermore that the HIC-aided turbo FD-DFE arrangement of Fig. 3 degenerates to the MMSE-SIC FD-DFE for $I=0$. Therefore, as observed in Fig. 5(c), the EXIT function of the PIC-aided turbo FD-DFE scheme becomes a two-segment curve, where the first sequent representing the L-MMSE is between the point $\left[\mathcal{I}_{\mathrm{A}}^{\mathrm{i}}, \mathcal{I}_{\mathrm{E}}^{\mathrm{i}}\right]=[0,0.67]$ and $\left[\mathcal{I}_{\mathrm{A}}^{\mathrm{i}}, \mathcal{I}_{\mathrm{E}}^{\mathrm{i}}\right]=[0.67,0.67]$, while the second segment representing the MF-PIC is between $\left[\mathcal{I}_{\mathrm{A}}^{\mathrm{i}}, \mathcal{I}_{\mathrm{E}}^{\mathrm{i}}\right]=[0.67,0.67]$ and $\left[\mathcal{I}_{\mathrm{A}}^{\mathrm{i}}, \mathcal{I}_{\mathrm{E}}^{\mathrm{i}}\right]=[1,0.91]$. Similarly for the HIC-aided turbo FD-DFE scheme, the corresponding segments in Fig. $5(\mathrm{e})$ are as follows: $[0,0.71]-[0.71,0.71] ;[0.71,0.71]-[1,0.91]$. Corresponding two-segment curves of L-MMSE and MMSE-SIC invoking BICM-2 are plotted in Fig. 5(d) and Fig. 5(f).

In the EXIT chart, the area below the inner component's EXIT curve, namely that of the turbo FDE, represents the maximum achievable rate of the transceiver. Additionally, the 'corner' points on these two-segment inner component EXIT curves are marked by the symbol $\star$ in Fig. 5(c) to Fig. 5(f). Turning our attention to the Monte-Carlo simulation based staircase-shaped decoding trajectories of the turbo FD-LE, PIC-aided turbo FD-DFE and HIC-aided turbo FD-DFE schemes, they are distinguished by symbols $\square, \triangle$ and $\diamond$, respectively, in all Fig. 5(a) to Fig. 5(f).

Observe in Fig. 5(a), Fig. 5(c) and Fig. 5(e) at a $E_{\mathrm{b}} / N_{0}=6.5$ $\mathrm{dB}$ that the turbo FD-LE has the highest maximum achievable throughput than the PIC-aided turbo FD-DFE and HIC-aided turbo FD-DFE, as indicated by its largest area below the inner component's curve. Furthermore, the $\mathcal{I}_{\mathrm{E}}^{\mathrm{i}}$ value of the MMSE-SIC FD-DFE inner component between $\mathcal{I}_{\mathrm{A}}^{\mathrm{i}}=0$ and $\mathcal{I}_{\mathrm{A}}^{\mathrm{i}}=0.93$ is higher than that of the L-MMSE curve. Based on a simular argument, the HIC-aided turbo FD-DFE exhibits a higher maximum achievable rate than the PIC-aided turbo FD-DFE, both of which relay on the same iterative MF-PIC. ${ }^{3}$ When BICM-2 aided system operating at the threshold of error-free decoding, the turbo FD-LE requires $I=12$ iterations at an $E_{\mathrm{b}} / N_{0}=4 \mathrm{~dB}$ in Fig. 5(b), while the PIC-aided and HIC-aided turbo FD-DFE only require $I=3$ iterations at an $E_{\mathrm{b}} / N_{0}=8 \mathrm{~dB}$ in Fig. 5(d) and Fig. 5(f), respectively.

The final BER performance curves upon varying the number of iterations $I$ are shown in Fig. 6, while the associated implementation complexity was quantified in Table II. Observe in Fig. 6(a) that all three types of turbo FDE schemes are capable of achieving a similar performance for $I=3$ in the BICM-1 aided SC-FDMA system. As seen in Fig. 6(b), albeit the turbo FD-LE provides a $2.5 \mathrm{~dB}$ power gain at a BER of $10^{-4}$ over the turbo FD-DFE scheme relying on $I=3$ iterations, the complexity of the turbo FD-LE is $\mathcal{O}\left\{N_{\mathrm{v}} C_{2}+C_{6}\right\}$ per

${ }^{2}$ The staircase-shaped black curves represent the actual Monte-Carlo simulation based mutual information exchange trajectories between the inner and outer components, which are indicated by the grey curves with labels. When operating without invoking iterations between the equaliser and FEC decoder for $I=0$, both the turbo FD-LE and PIC-aided turbo FD-DFE degenerate to a classic single-tap MMSE FD-LE.

${ }^{3}$ Naturally, at lower $E_{\mathrm{b}} / N_{0}$ values the inner component EXIT curves are shifted further down, indicating a lower achievable throughput, where the EXIT tunnel might become closed. 


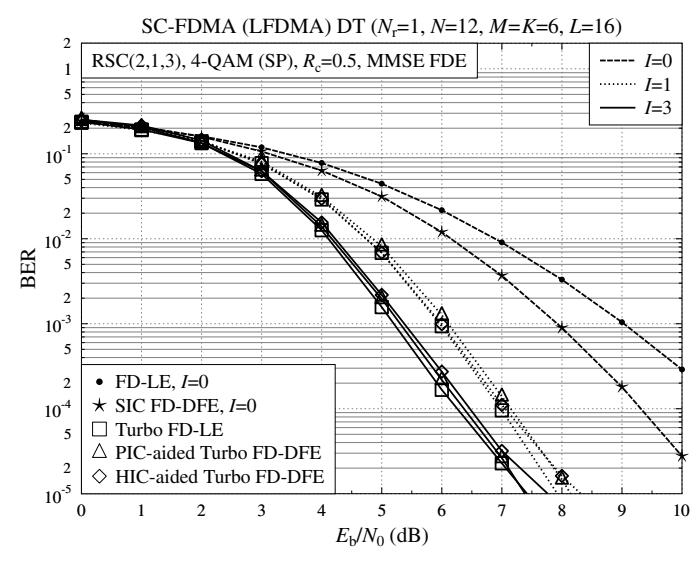

(a) Mode-1: 1/2-rate RSC coded 4-QAM

SC-FDMA (LFDMA) DT $\left(N_{\mathrm{r}}=1, N=12, M=K=6, L=16\right)$

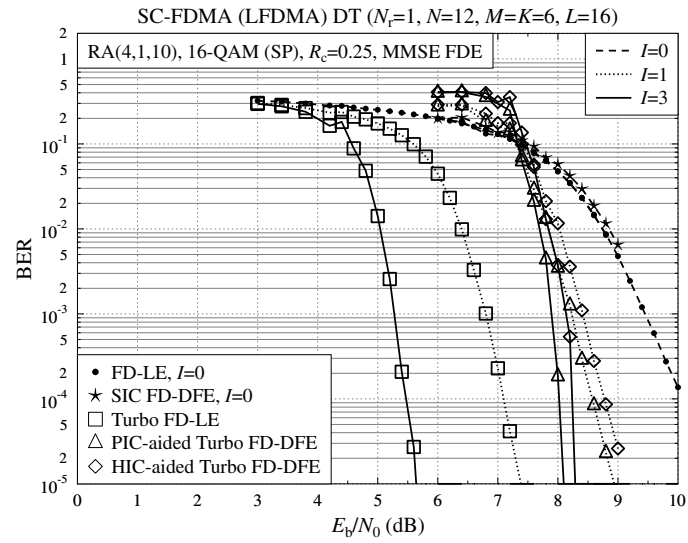

(b) Mode-2: 1/4-rate NS-IrRA coded 16-QAM

Fig. 6. BER performance of the various MMSE turbo FDEs schemes.

TABLE II

COMPLEXITIES OF VARIOUS TURBO FDES

\begin{tabular}{l|r}
\hline Algorithm & Complexity $C$ \\
\hline \hline 1: L-MMSE & $3 N^{3}+2 N^{2}+4 N-1$ \\
\hline 2: Soft-MMSE & $N^{4}+3 N^{3}+8 N^{2}+11 N$ \\
\hline 3: Real MMSE-SIC & $\left(8 \sqrt{2^{Q}}+5\right) N^{4}+\left(6 \sqrt{2^{Q}}+9\right) N^{3}+5 N^{2}$ \\
\hline 4: MF-PIC & $3 N^{3}+N^{2}-N$ \\
\hline 5: BICM decoding & $2^{Q} N_{\mathrm{v}}\left(2 N^{3}+N^{2}+2 Q N\right)+C_{\mathrm{D}}$ \\
\hline 6: BICM-ID per iter. & $2^{Q} N_{\mathrm{v}}\left[2 N^{3}+N^{2}+\left(Q^{2}+2 Q\right) N\right]+C_{\mathrm{E}}+C_{\mathrm{D}}$ \\
\hline \hline Turbo FD-LE & $\mathcal{O}\left\{N_{\mathrm{v}} C_{1}+C_{5}+I \cdot\left(N_{\mathrm{v}} C_{2}+C_{6}\right)\right\}$ \\
\hline PIC turbo FD-DFE & $\mathcal{O}\left\{N_{\mathrm{v}} C_{1}+C_{5}+I \cdot\left(N_{\mathrm{v}} C_{4}+C_{6}\right)\right\}$ \\
\hline HIC turbo FD-DFE & $\mathcal{O}\left\{N_{\mathrm{v}} C_{3}+C_{5}+I \cdot\left(N_{\mathrm{v}} C_{4}+C_{6}\right)\right\}$ \\
\hline
\end{tabular}

iteration for $Q$ bits per symbol, which is higher than the $\mathcal{O}\left\{N_{\mathrm{v}} C_{4}+\right.$ $\left.C_{6}\right\}$ per iteration for the PIC/HIC aided turbo FD-DFE schemes, as shown in Table II. ${ }^{4}$ Therefore, if the factor $N$ higher complexity per iteration of the FD-LE is not justifiable upon increasing $I$, then we advocate the low-complexity HIC-aided turbo FD-DFE for the BICM1 scenario and the low-complexity PIC-aided turbo FD-DFE for the BICM-2 assisted system. We note that, when the system invokes noniterative decoding, the complexity of the MMSE-SIC aided FD-DFE is a factor of $N$ higher than that of the L-MMSE aided FDE for a given $Q$.

${ }^{4}$ Observe in Table II, $C_{i},(i=1,2, \cdots, 6)$, are the complexities of listed algorithms for FDE and BICM, respectively, where the $C_{\mathrm{E}}$ and $C_{\mathrm{D}}$ denotes the complexities of FEC encoder and decoder as the outer component.

\section{CONCLUSIONS}

In this contribution, we investigated three types of turbo FDE receivers conceived for the LTE SC-FDMA uplink. Explicitly, a turbo FD-LE, a PIC-aided turbo FD-DFE and a HIC-aided turbo FD-DFE were conceived. Their performance was investigated with the aid of EXIT charts in the context of two BICM arrangements, when communicating over frequency-selective fading channels. Compared to the PIC-aided turbo FD-DFE, the HIC-aided one may provide a higher maximum rate. When the HIC-aided turbo FD-DFE associated with $I \geq 1$ iterations between the PIC-aided FD-DFE and FEC decoder, the advantage of the MMSE-SIC erodes, since the SIC was not incorporated into the iterative decoding loop. Our results demonstrated all the turbo FDEs considered are capable of achieve a similar BER performance, in conjunction with 1/2-rate RSC coded 4-QAM. By contrast, when the 1/4-rate NS-IrRA coded 16-QAM scheme is employed, the PIC-/HIC-aided turbo FD-DFEs may benefit from a low implementational complexity, while reducing the required transmission power by $0-3 \mathrm{~dB}$, in comparison to the turbo FD-LE. Additionally, when the 1/2-rate RSC coded 4-QAM system does not rely on iterative detection, the reliability-aided MMSE-SIC FD-DFE is capable of significantly outperforming the single-tap MMSE FDLE benchmark.

\section{REFERENCES}

[1] M. Tüchler and A. Singer, "Turbo equalization: An overview," IEEE Trans. Inf. Theory, vol. 57, no. 2, pp. 920-952, 2011.

[2] K. Kansanen and T. Matsumoto, "An analytical method for MMSE MIMO turbo equalizer EXIT chart computation," IEEE Trans. Wireless Commun., vol. 6, no. 1, pp. 59-63, Jan. 2007.

[3] L. Hanzo, T. Liew, B. Yeap, R. Tee, and S. Ng, Turbo Coding, Turbo Equalisation and Space-Time Coding (2nd Ed.). Wiley, 2011.

[4] F. Adachi, K. Takeda, and H. Tomeba, "Frequency-domain equalization for boradband single-carrier multiple access," IEICE Trans. Commun., vol. E92-B, no. 5, pp. 1441-1456, 2009.

[5] N. Benvenuto, R. Dinis, D. Falconer, and S. Tomasin, "Single carrier modulation with nonlinear frequency domain equalization: An idea whose time has come-again," Proc. IEEE, vol. 98, no. 1, pp. 69-96, Jan. 2010.

[6] D. Wang, X. Gao, and X. You, "Low complexity turbo receiver for multiuser STBC block transmission systems," IEEE Trans. Wireless Commun., vol. 5, no. 10, pp. 2625-2632, Oct. 2006.

[7] G. Berardinelli, B. Priyanto, T. Sorensen, and P. Mogensen, "Improving SC-FDMA performance by turbo equalization in UTRA LTE uplink," in Proc. IEEE VTC 2008-Spring, May 2008, pp. 2557-2561.

[8] C. Zhang, Z. Wang, C. Pan, S. Chen, and L. Hanzo, "Low-complexity iterative frequency domain decision feedback equalization," IEEE Trans. Veh. Technol., vol. 60, no. 3, pp. 1295-1301, Mar. 2011.

[9] B. Ng, C.-T. Lam, and D. Falconer, "Turbo frequency domain equalization for single-carrier broadband wireless systems," IEEE Trans. Wireless Commun., vol. 6, no. 2, pp. 759-767, Feb. 2007.

[10] T.-W. Yune, C.-H. Choi, G.-H. Im, and et. al., "SC-FDMA with iterative multiuser detection: improvements on power/spectral efficiency," IEEE Commun. Mag., vol. 48, no. 3, pp. 164-171, mar. 2010.

[11] M. Grossmann, "Outage performance analysis and code design for threestage MMSE turbo equalization in frequency-selective Rayleigh fading channels," IEEE Trans. Veh. Technol., vol. 60, no. 2, pp. 473-484, Feb. 2011.

[12] L.-L. Yang, "Receiver multiuser diversity aided multi-stage minimum mean-square error detection for heavily loaded DS-CDMA and SDMA systems," IEEE Trans. Commun., vol. 58, no. 12, pp. 3397-3404, Dec. 2010.

[13] $\_$, "Receiver multiuser diversity aided multi-stage MMSE multiuser detection for DS-CDMA and SDMA systems employing I-Q modulation," in Proc. IEEE VTC 2010-Fall, Sep. 2010, pp. 1-5.

[14] H. Jin, A. Khandekar, A. Kh, and R. J. McEliece, "Irregular repeat accumulate codes," in Proc. ICTC 2000, Munich, Germany, Sep. 2000, pp. $125-127$.

[15] L.-L. Yang, Multicarrier Communications. Wiley, 2009. 